or lighten it up to get a better idea of where the patient's at," says Librach.

Caution is particularly necessary when assessing a patient for continuous palliative sedation therapy to treat nonphysical suffering, says Blair Henry, a clinical ethicist at Sunnybrook Health Sciences Center in Toronto, Ontario, and a member of the team drafting the framework. "It's rare that such suffering would be called intolerable and refractory, but it's part of everyone's practice experience that you do occasionally meet people who are in such distress or crisis that no intervention of a psychologist or chaplain or any team member can relieve."

While such cases are most com- monly confused for physician-assisted suicide, withholding treatment, as a rule, would not be appropriate, says Librach. "I find it very difficult to separate out. In one case, I knew my patient was going to die a very difficult death with his bowel perforating but up to that point he wouldn't have much pain. He told me he was suffering waiting for the end, and I told him we would sedate him when the time came. We never were able to control his pain at end-oflife. He has this acute event, no one was standing by his bedside at the ready, and I've felt guilty ever since because he died a quick but very horrible, painful death."
Although patients regularly "walk away from the treatment table," refusing chemotherapy or dialysis, a patient's ability to "walk away from their sentience" is decidedly more limited by societal pressures, says Henry. "We're holding people from therapy they may in fact need because of value judgments we've made that a person should find meaning in their suffering."

The draft framework will be submitted to the Canadian Society of Palliative Care Physicians for review in the coming months. A final version will be published in 2011. — Lauren Vogel, CMAJ

CMAJ 2011. DOI:10.1503/cmaj.109-3766

\title{
Infectious risks in family doctors' offices
}

$\mathrm{W}$

hat a difference a year makes. During pandemic (H1N1) 2009, primary care providers were scurrying around offices and clinics removing books and toys from waiting rooms, positioning bottles of hand sanitizer, passing out surgical masks to patients who presented with a cough or fever and using hospital-grade disinfectant to mop up floors when a suspected H1N1 case left their office or clinic.

They were, of course, following infection control guidelines established and recommended by the College of Family Physicians (CFPC) and a number of other groups during the height of the pandemic.

As the threat fades into memory, there's an understandable tendency to hide the mops in the closet and leave the masks in the box.

But Ontario's top infectious disease doctor is urging primary care providers to maintain aggressive infection prevention and control measures in their practices year-round.

Returning to business-as-usual would be a mistake, says Dr. Doug Sider, acting director of infectious disease prevention and control for the Ontario Agency for Health Protection and Promotion. "To recommend that we only consider more aggressive compliance with all of these infection prevention and control measures in the face of something like pandemic influenza, and then step back, relax our vigilance, relax

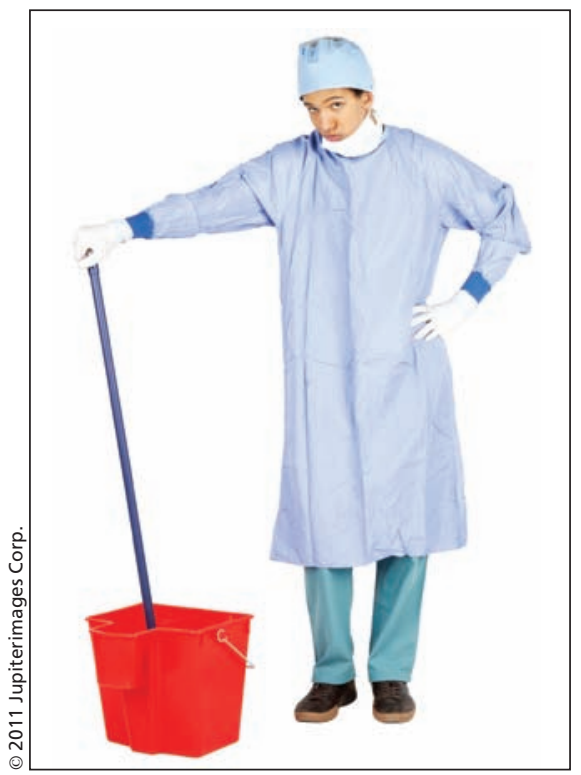

Keeping the mops moving is good infection control for practice, even though the public health threat posed by pandemic (H1N1) 2009 has abated.

our expectations, relax our urging to compliance at other times, just doesn't make a lot of sense to me."

The CFPC has never developed infection prevention and control guidelines for standard family practice. Although it was one of the associations responsible for developing and issuing the guidelines during the pandemic (along with the Canadian Medical Association, the National Specialty Society for Community Medicine and the Canadian Public Health Association), those do not apply outside of a pandemic, says Jayne Johnston, the college's communications manager.

The college doesn't have a general infection control policy telling members how they should, or should not, run their practices on a day-to-basis, Johnston says.

But some of those recommendations would be good practice year-round, particularly as the flu season descends, says Sider, who suggests that primary care doctors always ensure staff is immunized with the seasonal flu vaccine and that standardized procedures be adopted for hand hygiene and the conditions in which masks should be used.

Sider also urges regular screening for febrile respiratory illness and depending on office design, the adoption of measures to contain its spread, such as asking patients who present with a cough and fever to sit in one part of the waiting room. As an alternative, staff can use a telephone triage system to screen for respiratory illness and then cluster those appointments near the end of the day, he adds. That would limit transmission and allow for the office to be sanitized properly before the next business day.

Such measures are a challenge for many family practices, Sider acknowledges. "It's easy to say, but it's really hard to put into place. Hospitals have environmental services people that can 
orient themselves with a lot of resources and a lot of sophistication. What do you do with a solo GP? Or even a multi-group practice?"

Infection prevention and control in Canada has been focused on acute care facilities as a consequence of rising rates of health care-associated infections, which are now estimated at 220000 per year, according to a study conducted by members of the Canadian Hospital Epidemiology Committee, the Canadian Nosocomial Infection Surveillance Program and Health Canada.

While several provinces have created advisory committees and adopted measures aimed at reducing hospitalacquired infections and the Canadian Patient Safety Institute has rolled out a nationwide hand-hygiene campaign, most efforts have not been focused on health care facilities other than hospitals.

It's problematic, explains Dr. Mark Joffe, senior medical director of infection prevention and control for Alberta Health Services. For one, Alberta Health
Services can't tell a doctor how to run his or her private practice, Joffe says. And with limited resources, it makes sense to concentrate on areas in the health-care system where infection can do the most damage, which historically has meant a focus on acute care hospital settings. But Joffe says that increasingly, there are "more and more people involved with infection prevention and control in continuing care or nursing homes. That's a developing area."

As well, the Canadian Patient Safety Institutes will be bringing its "wash your hands, wash your hands, wash your hands" message to primary- and home-care providers across the country, says CEO Hugh MacLeod. It will also release the findings of a research project into patient safety in primary care and aim to change the culture by encouraging patients to ask doctors about infection prevention and control measures. "They [doctors] shouldn't be offended if someone says, 'have you washed your hands?'" MacLeod says.
"It's making everybody safe. It's in everybody's best interest."

While regulatory initiatives to implement infection prevention and control measures in the offices of family doctors are embryonic, at best, they are not entirely unheard of. The Alberta College of Physician and Surgeons, for example, has begun to conduct infection control audits of private practices.

Ward expects the college will proceed with some form of its infection prevention and control program in the coming months. "Infection prevention and control has not diminished in its importance, in the eye of the public, and the public health office and government. There is the ever-present threat of pandemic infections. We need to test everyone's readiness for those events. Will physicians' offices be safe places to visit during a pandemic? It will take a continuous educational campaign." — Emily Senger, Toronto, Ont.

CMAJ 2011. DOI:10.1503/cmaj.109-3748

\section{National home care standards urged}

$\mathrm{H}$ ome care has become one of the fastest-growing areas in Canadian health care over the past decade, though, as is often the case in Canada's fractured health care landscape, some provinces are performing better than others.

It is difficult, however, to compare the state of home care in different jurisdictions because the very notion of home care differs from province to province. This is why, according to Marg McAlister, a project manager for the Canadian Home Care Association, Canada needs a set of national standards for home care.

"It is hard to compare province to province because each defines the roles of various professions in home care differently," says McAlister.

The most comprehensive look at home care across Canada can be found in the Canadian Home Care Association's Portraits of Home Care in Canada 2008 (www.cdnhomecare.ca/media.php ?mid=1877), an update of a 2003 report that gave home care leaders the opportunity to "have their voices heard through their descriptions of home care as it is known and understood within each of their respective jurisdictions." Though the document warns that "valid comparisons cannot be made because of the

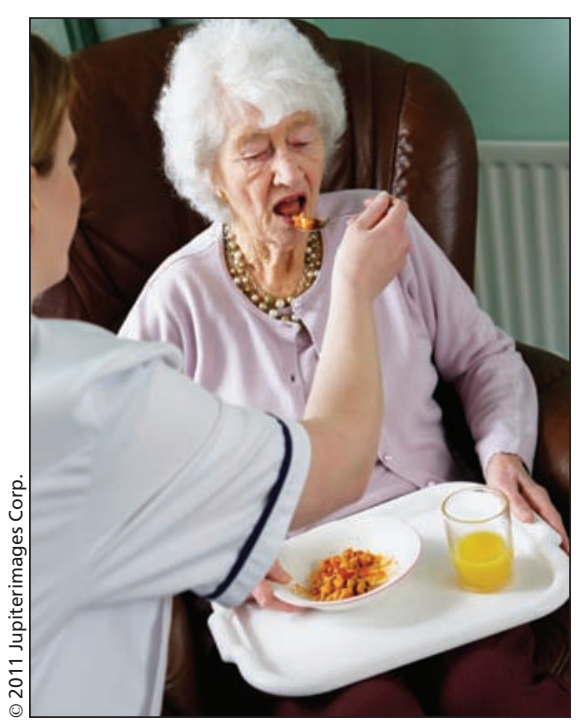

There is a significant nation-wide variation in the maximum number of hours per week of home care services that people are entitled to access. absence of data definitions and the variation of data collection and reporting across Canada," it does offer "snapshots" of home care programs, which highlight such features as governance, organization, services, quality and accountability (Table 1).

With respect to governance, there is little variation across the country, with 12 of 13 provinces and territories operating home care services under the jurisdiction of their ministries of health. The lone exception is New Brunswick, where home care is run by the New Brunswick Department of Health and Wellness and the New Brunswick Department of Social Development.

Legislation for home care, on the other hand, varies widely - tucked into various acts, orders-in-council, guidelines and policies. "This lack of a specific legislative framework for home care contributes to the wide variation in access and availability of services across Canada," the report states.

In terms of services, almost all jurisdictions offer core services such as case 